\section{El apoyo activo como herramienta para la mejora de la participación de la persona con discapacidad intelectual. Estado de la cuestión}

\author{
Active support as a tool for improving the \\ participation of people with intellectual \\ disabilities. State of the art
}

\section{Resumen}

El apoyo activo, a pesar de ser utilizado desde hace más de 20 años en otros lugares, aún es una metodología desconocida en los servicios que prestan apoyo a las personas con discapacidad intelectual y altas necesidades de apoyo en nuestro país. En este artículo se pretende realizar un acercamiento a la fundamentación y a las estrategias promulgadas por el apoyo activo, a través de una revisión documental de 24 estudios en los que se analizan los cambios observados, tanto en la vida de los residentes como en la actividad de los profesionales de apoyo directo tras la aplicación de esta metodología.

\section{Palabras clave}

Apoyo activo, discapacidad intelectual, necesidades de apoyo, revisión documental.

\begin{abstract}
Active support, despite being used for more than 20 years in other places, it is still an unknown methodology in services that support people with intellectual disabilities and high support needs, in our country. This article aims to make an approach to the philosophy and strategies promulgated by the active support, through a documental review of scientific studies. Results of 24 articles about the life of residents and the direct support professionals' work following the implementation of this methodology in different resources are analyzed.
\end{abstract}

\section{Keywords}

Active support, intellectual disability, support needs, literature review.

\section{Tania Cuervo Rodríguez \\ <uor94650@uniovi.es> \\ Universidad de Oviedo}

María Teresa Iglesias García <teresai@uniovi.es>

Universidad de Oviedo

\section{Samuel Fernández \\ Fernández \\ <samuel@uniovi.es> \\ Universidad de Oviedo}

Para citar:

Cuervo, T. et al. (2016): "El apoyo activo como herramienta para la mejora de la participación de la persona con discapacidad intelectual. Estado de la cuestión". Revista Española de Discapacidad, 4 (2): 47-62.

Doi: <https://doi.org/ıO.5569/2340$5 \mathrm{IO} 4.04 .02 .03>$

Fecha de recepción: 08-04-20I6 Fecha de aceptación: I4-06-20I6 


\section{Introducción}

Tradicionalmente, los servicios destinados a personas con discapacidad intelectual han ofrecido a sus usuarios un modelo de asistencia que se ha denominado 'modo hotel', en el cuál los profesionales hacen todo 'por' la persona en vez de 'con' ella (Association for Real Change, 2009). Como consecuencia de esta forma de actuar, la persona pasa la mayor parte de su tiempo inactiva, tal y como se ha visto en diferentes estudios, como el de Emmerson et al. (2000), en el que determinaron que el tiempo que la persona estaba acompañada era de I 2 minutos por cada hora. En cambio, las metodologías desarrolladas en las últimas décadas proponen que la persona tenga una mayor participación en su vida diaria.

Jones et al. (20I I) describen cuáles son los aspectos importantes para todos los individuos en su vida diaria, independientemente de sus necesidades de apoyo: participación comunitaria, establecimiento de relaciones sociales y personales, posibilidad de vivir nuevas experiencias, tomar elecciones, tener el control de su vida, tener un estatus y ser tratado como una persona. Todas ellas deberían ser las finalidades principales de cualquier actuación llevada a cabo por las instituciones.

El apoyo activo (de aquí en adelante AA), surge en el Reino Unido en los años 80 (Totsika et al., 2008; Mansell y Beadle-Brown, 2OI2) con el objetivo de que la asistencia que reciben las personas con discapacidad intelectual les permita una mayor participación (Cerolini y Ferrie, 2005; Stancliffe et al., 2005) y mejorar los aspectos descritos anteriormente por Jones et al. (20I I). Su primer desarrollo tuvo lugar en un grupo de residencias inglesas en las que se observó que, gracias al AA, los profesionales prestaban una atención más eficaz, entendiendo por ello la participación en actividades significativas por parte de las personas con discapacidad intelectual (Stancliffe et al. 2005).
Elementos característicos del apoyo activo

Esta metodología presenta, en su actual estado de desarrollo, la siguiente fundamentación (Cerolini y Ferrie, 2005; Mansell et al., 2005; Totsika et al., 2008; Jones et al., 20I I; Mansell y Beadle-Brown, 20I2):

- Los profesionales necesitan conocer bien a la persona a la que se asiste para que la atención sea más eficaz.

- El trabajador no puede hacer todo por la persona.

- Es necesario hacer un seguimiento de las oportunidades de participación que tiene la persona con discapacidad cada día, siempre basado en las evidencias.

- $\quad$ El feedback entre profesionales es primordial para que los apoyos estén coordinados.

- $\quad$ El feedback con las familias también es importante para que estén más implicados.

- La organización ha de tener contacto con la comunidad en la que está ya que proporciona a la persona mayores oportunidades de participación.

- Las rutinas diarias han de ser flexibles.

- Los profesionales han de procurar que las actividades en las que participan las personas con discapacidad sean variadas y significativas.

- Se potencia que la persona establezca y mantenga relaciones personales con sus familiares y amigos.

- Todos los momentos del día tienen potencial para que la persona participe.

- Es mejor hacer pocas actividades y más a menudo.

- Las instituciones han de garantizar que la persona pueda realizar elecciones y tener un mayor control de su vida.

En la tabla I se exponen brevemente las principales estrategias del apoyo activo para mejorar la vida de las personas con discapacidad: 


Tabla 1. Estrategias del apoyo activo
\begin{tabular}{|l|l|}
\hline Estrategia & Características \\
\hline Protocolos de actividad & $\begin{array}{l}\text { En ellos se describen las actividades que se realizan más frecuentemente. } \\
\text { Cada una de ellas se subdividirá en otras más pequeñas para que resulten } \\
\text { más fáciles para la persona. }\end{array}$ \\
\hline Planes de apoyo a la actividad & $\begin{array}{l}\text { Tienen el formato de una hoja por día, en la que se describirán las } \\
\text { actividades, el apoyo que necesita la persona y el seguimiento que } \\
\text { se realizará a lo largo del día. Si la persona lo requiere, se utilizarán } \\
\text { pictogramas para que sea accesible. }\end{array}$ \\
\hline Planes individuales & $\begin{array}{l}\text { En este documento se expone, de manera individualizada, las actuaciones } \\
\text { a medio plazo con los resultados prioritarios esperados en cada una de } \\
\text { ellas. }\end{array}$ \\
\hline Planes de oportunidad & $\begin{array}{l}\text { Estos planes recogen las ocasiones en las que la persona tiene la } \\
\text { oportunidad de participar, con el objetivo de crear contextos en los que } \\
\text { pueda desarrollar sus propias habilidades a través de la realización de } \\
\text { diversas actividades de forma regular. }\end{array}$ \\
\hline $\begin{array}{l}\text { Planes de enseñanza } \\
\text { estructurados }\end{array}$ & $\begin{array}{l}\text { En este tipo de documentos se recogen objetivos de participación pero a } \\
\text { largo plazo a través de una enseñanza más estructurada. }\end{array}$ \\
\hline
\end{tabular}

Fuente: Totsika et al., 2008b.

A la hora de crear estos planes de apoyo activo, se han de tener en cuenta las siguientes premisas (Jones et al., 20II):

I. A la hora de planificar las actividades de la vida diaria, es necesario que haya una buena coordinación con el resto de personas de la entidad.

2. Estos planes ayudan a que la persona sea más autónoma.

3. Los planes han de presentarse por escrito para que haya una mejor coordinación entre los profesionales.

4. Estos planes incluyen: actividades domésticas, actividades personales y de autocuidado, actividades de tiempo libre, actividades vocacionales y actividades de carácter social.

5. Al finalizar cada día, los profesionales se reunirán para revisar si se ha conseguido la participación en la actividad prevista.

6. La familia debe de incluirse a la hora de decidir sobre alguna actividad cuando la persona no pueda expresarse por sí misma.

7. Estos planes no reemplazarán a los diarios personales ya que se utilizarán en conjunto.

8. Para facilitar la comprensión de estos documentos, los planes pueden hacerse a través de imágenes, objetos... o cualquier elemento que sea accesible para la persona con discapacidad.

9. Los profesionales decidirán acerca de qué actividad de la vida diaria se realizará cada día y qué trabajador o trabajadores proporcionará el apoyo necesario a la persona.

Respecto a la forma de dar este apoyo, existen diversas alternativas en función de las necesidades de la persona: se puede sugerir, pedir o decir (propuesta verbal para hacer consciente a la persona de que es el momento para hacer una determinada cosa), dar instrucciones (también tiene un carácter verbal pero se guía a la persona sobre lo que tiene que hacer en cada momento), hacer un gesto (lo cual funciona bien cuando no se comprenden las instrucciones verbales), o mostrar cómo se hace una cosa o guiar, es decir, dar directamente el apoyo físico que se necesite (Jones et al., 20I I).

El modelo de 5 puntos de O’Brien es básico a la hora de formar a los profesionales acerca de cómo trabajar con las personas con discapacidad mediante la metodología del apoyo activo. Este modelo recoge las siguientes ideas (Mansell y Beadle-Brown, 20I2): 
I. Presencia: es el nivel en el que la persona es el centro de los acuerdos que se toman respecto a sus actividades de la vida diaria, de trabajo y de ocio.

2. Elección: hace referencia a la autonomía que tiene la persona con discapacidad a la hora de tomar decisiones acerca de su vida.

3. Competencia: es el nivel de experiencias que ha vivido la persona a lo largo de su vida y la variedad de actividades que le puede ofrecer la institución para ayudar a la persona.

4. Participación: la variedad y el alcance de las relaciones que tiene la persona.

5. Respeto: ha de ser valorado como cualquier otro miembro del grupo.

Pero en ocasiones existen problemas a la hora de instaurar el AA a los que las organizaciones han de hacer frente y buscar la solución adecuada en beneficio tanto de las personas con discapacidad como de los propios profesionales. Algunas de las barreras más comunes que pueden encontrarse son: algunos trabajadores no ven la importancia de la participación de la persona con discapacidad en las actividades de su vida diaria y consideran que hay otras cosas que tienen más importancia, otros se centran más en su labor burocrática que en la calidad de los apoyos que está proporcionando, en ocasiones se deciden las actividades en las que participará la persona en función de los intereses del personal en vez de los de la propia persona, y suelen seleccionarse únicamente actividades domésticas relegando a un segundo plano otro tipo de actividades (Mansell y Beadle-Brown, 20I2).

Desde la implantación de esta metodología, han sido varios los estudios que tienen como finalidad analizar los beneficios del apoyo activo. Es por ello que este artículo tiene como objetivo principal el análisis de sus posibles aportaciones. A continuación se describe el proceso de búsqueda que se ha seguido para llegar a los 24 artículos que componen esta revisión documental, los resultados obtenidos (tanto los beneficios para los profesionales como para las personas con discapacidad) y las conclusiones a las que se ha llegado tras la realización de este proceso.

\section{Proceso de búsqueda}

En el gráfico I se puede observar cuál ha sido el proceso de búsqueda realizado para obtener los artículos que conforman esta revisión documental. En primer lugar, se han consultado las siguientes bases de datos del ámbito nacional e internacional: Latindex, DOAJ, Redib, Dulcinea, Dialnet, ERIH PLUS, ISOC, DICE, SciELO y MIAR. A su vez, se ha utilizado un listado de revistas académicas del ámbito de la discapacidad elaborado por la Universidad de Galway, Irlanda, en noviembre de 2013 (Nui Galway, 2013). Todas estas bases han sido consultadas entre el i 5 de enero y el I de febrero de 2016.

En cada una de las bases de datos citadas se han utilizado los descriptores de 'discapacidad', 'discapacidad intelectual', 'disability', 'learning disability’ e 'intellectual disability’, dando como resultado 3 I revistas cuya temática se relacionaba con el objetivo de este estudio. Posteriormente, se ha procedido a buscar en estas revistas el descriptor 'active support', lo que ha proporcionado 267 artículos, de los cuáles, tal y como se refleja en la tabla 2 , se han seleccionado únicamente 24 pertenecientes a 6 revistas.

El motivo de la selección de estos documentos ha sido que, una vez leídos los abstracts, se trataban de investigaciones cuya principal finalidad es analizar los beneficios de la implantación del apoyo activo en centros que atienden a personas con discapacidad intelectual. El Io०\% se trata de literatura inglesa (la mayoría anglosajona), y no se ha encontrado ningún artículo en lengua castellana.

\section{Resultados}

Tal y como se ha indicado en los apartados anteriores, en general, el objetivo de todos los artículos seleccionados es el de analizar si la 


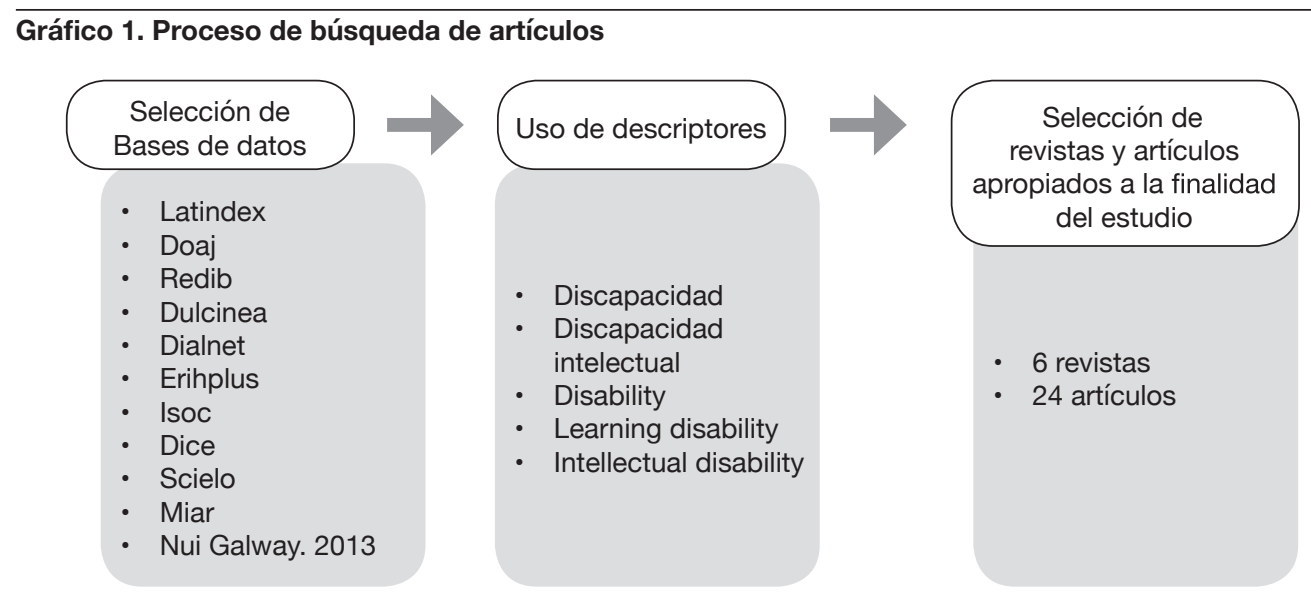

Fuente: elaboración propia.

\begin{tabular}{l}
\hline Tabla 2. Resumen de artículos y revistas seleccionadas en este estudio \\
\begin{tabular}{|l|c|}
\hline Revista & No de Artículos \\
\hline Research in Developmental Disabilities & 3 \\
\hline Journal of Intellectual Disability Research & 3 \\
\hline American Journal of Intellectual and Developmental Disabilities & 1 \\
\hline Journal of Applied Research in Intellectual Disabilities & 7 \\
\hline Tizard Learning Disability Review & \\
\hline Journal of Intellectual and Developmental Disability & 3 \\
\hline
\end{tabular} \\
\hline
\end{tabular}

Fuente: elaboración propia.

implantación del apoyo activo tiene un impacto positivo en los diferentes niveles de la vida de las personas con discapacidad intelectual y en las prácticas de atención ejercidas por los diferentes profesionales. A pesar de que las muestras utilizadas son muy diferentes en cada uno de los estudios (el número de residentes oscila entre
I y 359 , el número de profesionales entre 3 y 354, y el número de hogares entre 5 y I 38 ), todos ellos muestran los beneficios del apoyo activo (ver tabla 3), para lo que ha utilizado una extensa variedad de instrumentos de recogida de información, los cuáles han sido resumidos en la tabla 4 . 


\begin{tabular}{|c|c|c|c|}
\hline Autores & Año & Objetivo principal & Muestra \\
\hline Jones et al. & 1999 & $\begin{array}{l}\text { Analizar los efectos en los residentes de la formación } \\
\text { en AA del personal. }\end{array}$ & $\begin{array}{l}5 \text { hogares } \\
19 \text { residentes } \\
\end{array}$ \\
\hline Felce et al. & 2000 & $\begin{array}{l}\text { Analizar los beneficios del AA en los hogares } \\
\text { participantes tras la formación del personal. }\end{array}$ & $\begin{array}{l}5 \text { hogares } \\
19 \text { residentes } \\
52 \text { profesionales }\end{array}$ \\
\hline Jones et al. & $2001 a$ & $\begin{array}{l}\text { Analizar los efectos en los residentes y en la } \\
\text { asistencia que reciben tras la formación en AA del } \\
\text { personal. }\end{array}$ & $\begin{array}{l}38 \text { hogares } \\
106 \text { residentes } \\
303 \text { profesionales }\end{array}$ \\
\hline Jones et al. & $2001 b$ & $\begin{array}{l}\text { Conocer los cambios en la asistencia que reciben los } \\
\text { residentes y los efectos en diversos aspectos de su } \\
\text { vida tras la formación del personal en AA. }\end{array}$ & $\begin{array}{l}74 \text { hogares } \\
188 \text { residentes } \\
\end{array}$ \\
\hline Smith et al. & 2002 & $\begin{array}{l}\text { Analizar la eficacia de los apoyos que proporcionan } \\
\text { los profesionales tras su formación en AA. }\end{array}$ & $\begin{array}{l}74 \text { hogares } \\
188 \text { residentes } \\
\end{array}$ \\
\hline Mansell et al. & 2002 & $\begin{array}{l}\text { Analizar los efectos de la implantación del AA tres } \\
\text { años después del primer estudio realizado por los } \\
\text { mismos autores. }\end{array}$ & $\begin{array}{l}\text { Primer estudio } \\
13 \text { hogares } \\
26 \text { residentes } \\
\\
\text { Segundo estudio } \\
13 \text { hogares } \\
23 \text { residentes }\end{array}$ \\
\hline Bradshaw et al. & 2004 & $\begin{array}{l}\text { Analizar los efectos de la formación en AA del } \\
\text { personal en diversos aspectos de la vida de los } \\
\text { residentes. }\end{array}$ & $\begin{array}{l}3 \text { hogares } \\
38 \text { profesionales }\end{array}$ \\
\hline Stancliffe et al. & 2007 & $\begin{array}{l}\text { Analizar si el AA resulta adecuado para las personas } \\
\text { con mayores niveles de participación y si hay } \\
\text { cambios tras la formación del personal en AA. }\end{array}$ & $\begin{array}{l}5 \text { hogares } \\
22 \text { residentes } \\
31 \text { profesionales } \\
5 \text { managers } \\
\end{array}$ \\
\hline Mansell et al. & 2008 & $\begin{array}{l}\text { Analizar los efectos en los residentes y en la atención } \\
\text { que reciben tras la formación del personal en AA, y } \\
\text { compararlo con un grupo de control. }\end{array}$ & \begin{tabular}{|l}
72 hogares \\
359 residentes \\
354 profesionales \\
\end{tabular} \\
\hline Fyffe et al. & 2008 & $\begin{array}{l}\text { Analizar si existe relación entre el cambio de las } \\
\text { prácticas de los profesionales y la aplicación del AA. }\end{array}$ & \begin{tabular}{|l|}
11 hogares \\
11 supervisores \\
53 profesionales \\
16 managers de "non- \\
house-based" \\
\end{tabular} \\
\hline Koritsas et al. & 2008 & $\begin{array}{l}\text { Analizar los cambios en diferentes áreas de la vida de } \\
\text { los residentes tras la formación de los profesionales } \\
\text { en AA. }\end{array}$ & $\begin{array}{l}3 \text { hogares } \\
12 \text { residentes } \\
11 \text { profesionales }\end{array}$ \\
\hline Stancliffe et al. & 2008 & $\begin{array}{l}\text { Examinar, tras la formación en AA, el tiempo que los } \\
\text { profesionales ayudan a los residentes y la cantidad } \\
\text { de elogios que les proporcionan para mejorar su } \\
\text { participación. }\end{array}$ & $\begin{array}{l}22 \text { residentes } \\
31 \text { profesionales } \\
5 \text { managers }\end{array}$ \\
\hline $\begin{array}{l}\text { Beadle-Brown } \\
\text { et al. }\end{array}$ & 2008 & $\begin{array}{l}\text { Analizar los beneficios para los residentes y para el } \\
\text { personal tras la formación en AA. }\end{array}$ & $\begin{array}{l}6 \text { hogares } \\
29 \text { residentes } \\
\end{array}$ \\
\hline Totsika et al. & $2008 \mathrm{a}$ & $\begin{array}{l}\text { Analizar la visión que tiene el personal sobre la } \\
\text { formación que han recibido sobre AA y los beneficios } \\
\text { que les ha reportado en sus prácticas de apoyo. }\end{array}$ & $\begin{array}{l}10 \text { hogares } \\
20 \text { residentes } \\
58 \text { profesionales }\end{array}$ \\
\hline Toogood et al. & 2009 & $\begin{array}{l}\text { Analizar los cambios en el comportamiento de un } \\
\text { residente utilizando procesos del AA. }\end{array}$ & 1 residente \\
\hline
\end{tabular}




\begin{tabular}{|c|c|c|c|}
\hline Autores & Año & Objetivo principal & Muestra \\
\hline Stancliffe et al. & 2010 & $\begin{array}{l}\text { Analizar los cambios en participación doméstica, } \\
\text { conducta adaptativa, cambios de comportamiento y } \\
\text { depresión, en los residentes seis meses después de } \\
\text { la implementación del AA. }\end{array}$ & $\begin{array}{l}9 \text { hogares } \\
41 \text { residentes }\end{array}$ \\
\hline Totsika et al. & 2010 & $\begin{array}{l}\text { Analizar la efectividad del "entrenamiento interactivo" } \\
\text { en AA en la mejora de la vida de los residentes. }\end{array}$ & $\begin{array}{l}10 \text { hogares } \\
21 \text { residentes }\end{array}$ \\
\hline Chou et al. & 2011 & $\begin{array}{l}\text { Demostrar que el AA supone un beneficio para } \\
\text { los residentes en cuanto a su participación en } \\
\text { actividades, en relaciones sociales, en elecciones y } \\
\text { en conductas adaptativas, a la vez que reduce sus } \\
\text { niveles de depresión. }\end{array}$ & $\begin{array}{l}\text { Antes del pre-test } \\
13 \text { supervisores } \\
62 \text { residentes } \\
\\
\text { Después del pre-test } \\
12 \text { hogares } \\
49 \text { residentes } \\
4 \text { supervisores } \\
6 \text { managers }\end{array}$ \\
\hline Stancliffe et al. & 2011 & $\begin{array}{l}\text { Analizar, tras la formación del personal en } \mathrm{AA} \text {, } \\
\text { la efectividad de aumentar de uno a dos los } \\
\text { profesionales que trabajan con cada residente. }\end{array}$ & $\begin{array}{l}1 \text { hogar } \\
4 \text { residentes } \\
1 \text { manager } \\
7 \text { profesionales }\end{array}$ \\
\hline Riches et al. & 2011 & $\begin{array}{l}\text { Analizar los beneficios para los residentes y para los } \\
\text { profesionales tras la formación en AA. }\end{array}$ & $\begin{array}{l}\text { Grupo } 1 \\
6 \text { hogares } \\
22 \text { residentes } \\
65 \text { profesionales } \\
\text { Grupo } 2 \\
6 \text { hogares } \\
23 \text { residentes } \\
54 \text { profesionales }\end{array}$ \\
\hline $\begin{array}{l}\text { Beadle-Brown } \\
\text { et al. }\end{array}$ & 2012 & $\begin{array}{l}\text { Analizar los beneficios para los residentes y para el } \\
\text { personal, un año después de la formación en AA. }\end{array}$ & $\begin{array}{l}6 \text { hogares } \\
31 \text { residentes }\end{array}$ \\
\hline Graham et al. & 2013 & $\begin{array}{l}\text { Analizar los beneficios del AA en los residentes y en } \\
\text { el apoyo que proporcionan los profesionales, dos } \\
\text { años y medio después de su implementación. }\end{array}$ & $\begin{array}{l}4 \text { hogares } \\
4 \text { residentes } \\
3 \text { familias } \\
4 \text { managers } \\
3 \text { profesionales de la salud }\end{array}$ \\
\hline Mansell et al. & 2013 & $\begin{array}{l}\text { Analizar, diez años después de la implantación del } \\
\text { AA, los principios del apoyo activo que utilizan los } \\
\text { profesionales y en qué medida se ofrece realmente } \\
\text { esta metodología a los residentes. }\end{array}$ & $\begin{array}{l}34 \text { hogares } \\
151 \text { residentes }\end{array}$ \\
\hline $\begin{array}{l}\text { Beadle-Brown } \\
\text { et al. }\end{array}$ & 2014 & $\begin{array}{l}\text { Explorar el papel del liderazgo en los hogares de } \\
\text { personas con discapacidad intelectual y conocer su } \\
\text { relación con la variación del uso del AA. }\end{array}$ & $\begin{array}{l}2005 / 2006 \\
138 \text { hogares } \\
649 \text { personas } \\
\\
2009 \\
33 \text { hogares } \\
126 \text { residentes } \\
\\
2010 \\
39 \text { hogares } \\
124 \text { residentes }\end{array}$ \\
\hline
\end{tabular}

Fuente: elaboración propia. 
Tabla 4. Instrumentos de recogida de información utilizados

\begin{tabular}{|c|c|c|}
\hline Instrumento & $\begin{array}{l}N^{\circ} \text { de Artículos } \\
\text { que lo utiliza }\end{array}$ & $\begin{array}{c}\% \text { de Artículos que lo } \\
\text { utiliza }\end{array}$ \\
\hline Aberrant Behavior Checklist & 7 & $29,1 \%$ \\
\hline Active Support Measure & 6 & $25 \%$ \\
\hline Adaptive Support Checklist & 1 & $4,1 \%$ \\
\hline Adaptive Behavior Scale & 10 & $41,6 \%$ \\
\hline Assessment Schedule & 1 & $4,1 \%$ \\
\hline Behavior Problems Inventory & 1 & $4,1 \%$ \\
\hline Choice Making Scale & 1 & $4,1 \%$ \\
\hline Choice Questionnaire & 3 & $12,5 \%$ \\
\hline Cuestionario elaborado por los investigadores & 1 & $4,1 \%$ \\
\hline Depression Scale & 2 & $8,3 \%$ \\
\hline Developmental Behavior Checklist for Adults & 1 & $4,1 \%$ \\
\hline Disability Assessment Schedule & 1 & $4,1 \%$ \\
\hline Entrevistas a profesionales & 5 & $20,8 \%$ \\
\hline Feedback Questionnaire & 1 & $4,1 \%$ \\
\hline Group Home Management Scale & 2 & $8,3 \%$ \\
\hline Grupos de enfoque & 1 & $4,1 \%$ \\
\hline Index of Community Involvement Revised & 4 & $16,6 \%$ \\
\hline Index of Participation in Daily Living & 1 & $4,1 \%$ \\
\hline Index of Participation in Domestic Life & 6 & $25 \%$ \\
\hline Información proporcionada por el centro & 3 & $12,5 \%$ \\
\hline Inventory for Client and Agency Planning & 5 & $20,8 \%$ \\
\hline Monetary Time Sampling & 1 & $4,1 \%$ \\
\hline Mood Scale & 1 & $4,1 \%$ \\
\hline Observación & 16 & $66,6 \%$ \\
\hline Psychopathology Instrument for Mentally Retarded Adults & 2 & $8,3 \%$ \\
\hline Quality of Management Scale & 1 & $4,1 \%$ \\
\hline Residential Services Working Practices Scale & 3 & $12,5 \%$ \\
\hline Revised Residential Services Settings Questionnaire & 3 & $12,5 \%$ \\
\hline Schedule of Handicaps Behavior and Skills & 3 & $12,5 \%$ \\
\hline Short User Survey & 2 & $8,3 \%$ \\
\hline Social Network Index & 2 & $8,3 \%$ \\
\hline Staff Experience and Satisfaction Questionnaire & 3 & $12,5 \%$ \\
\hline Staffing information Interview & 3 & $12,5 \%$ \\
\hline Supports Intensity Scale & 1 & $4,1 \%$ \\
\hline
\end{tabular}

Fuente: elaboración propia. 
A continuación se detallan los resultados obtenidos en esta revisión organizados en tres grupos: formación en apoyo activo, resultados para los profesionales y resultados para las personas con discapacidad (en las áreas de participación, interacción social, depresión y conducta adaptativa y cambios de comportamiento).

\section{- Formación en apoyo activo}

Todas las investigaciones en las que se forma al personal en apoyo activo combinan una parte de workshop, que incluye una formación teórica (normalmente de uno o dos días) en las que se exponen conocimientos relativos a qué es el AA, cuáles son sus planteamientos, qué estrategias sigue..., y una parte de entrenamiento interactivo, práctico, en el propio lugar de trabajo.

En la mayor parte de los estudios analizados, la persona encargada de formar a los profesionales en AA es un experto perteneciente a una entidad externa, por lo que la parte práctica se hace más compleja ya que en ocasiones no conoce a los residentes. En la investigación realizada por Totsika et al. (2008a), en la que se analizaba la efectividad del entrenamiento interactivo como parte de la formación de los trabajadores, más de la mitad de los encuestados (53\%) que recibieron esta modalidad de formación planteaban que ésta hubiese sido más efectiva si fuese desarrollada por alguien que conozca previamente a las personas con discapacidad.

Tras la formación completa en apoyo activo, los profesionales reportan: una mayor conciencia sobre las necesidades de los residentes y haber cambiado su forma de trabajo (Totsika et al., 2008a), la adquisición de nuevas habilidades, herramientas y técnicas para proporcionar mejores apoyos acordes con las necesidades de las personas con discapacidad intelectual (Smith et al., 2002; Totsika et al., 2008a; Riches et al., 20I I; Graham et al., 20I3), y, en general, una satisfacción con el proceso de aprendizaje del apoyo activo (Totsika et al., 2008a; Riches et al., 2OII).
Por lo tanto, la combinación del workshop teórico con el entrenamiento interactivo supone una metodología de formación positiva para que los profesionales puedan prestar una asistencia más efectiva a las personas con discapacidades más severas (Smith et al., 2002; Totsika et al., 20I0).

\section{- Resultados para los profesionales}

Los cambios propiciados en las prácticas de apoyo de los profesionales hacia los residentes suponen un cambio también en la vida de las propias personas con discapacidad: al recibir una mayor atención se ven mejoradas, a su vez, muchas dimensiones de su calidad de vida.

Diversos estudios informan de un aumento significativo de la ayuda que el personal proporciona a los usuarios del servicio (Stancliffe et al., 2007). En uno de ellos este cambio es más amplio ya que pasa a ser del $2 \%$ al $35 \%$ (Jones et al., I999), mientras que en otros el cambio no es tan grande, como en el estudio de Stancliffe et al. (20I I), en el que la asistencia aumentó del $2 \mathrm{I}, 5 \%$ al 35,7\% con la presencia de dos profesionales en vez de uno.

En consonancia con este cambio, también se produce una mejora en el contacto por parte del personal, siendo, por ejemplo, antes de la formación en AA del I 6,7 \% y posteriormente del $2 \mathrm{I}, 2 \%$ en una de las investigaciones (Bradshaw et al., 2004), o del $54 \%$ en el comienzo del estudio y del $59 \%$ al final en otro de los artículos analizados (Riches et al., 20II). A pesar de producirse este aumento, la correlación entre el contacto del personal y la participación de los residentes no es estadísticamente significativa según los resultados obtenidos en el estudio de Bradshaw et al. (2004). Por otro lado, Stancliffe et al. (2007) asocian significativamente ese incremento de ayuda recibida con el aumento en la participación de las personas con discapacidad.

La asistencia no verbal como estrategia de apoyo al residente ha cambiado a lo largo del proceso, adaptándose a las necesidades de 
cada una de las personas con discapacidad a las que se atendía (Smith et al., 2002). A pesar de ello, la investigación realizada por Totsika et al. (2010) recoge que estos cambios no son significativos.

En relación a los cambios organizacionales, es destacable que las prácticas de liderazgo de los profesionales también han incrementado tras la implantación del apoyo activo (Beadle- Brown et al., 2008; 20I 2). Por ejemplo, Beadle-Brown et al. (2OI4) reportan un aumento del $42 \%$ al $52 \%$, lo que acarrea como consecuencia mejores y mayores resultados en la adopción de las estrategias del AA. Por otra parte, Riches et al., (20II) reportan una reducción de las actividades burocráticas del $46 \%$ al $4 \mathrm{I} \%$, permitiendo que los profesionales puedan dedicar ese tiempo a la atención directa a los residentes.

El trabajo en equipo y coordinado es uno de los factores clave para que los apoyos que requieren los usuarios del servicio sean de calidad y efectivos. En este aspecto se han producido mejoras en la comunicación interprofesional y en sus habilidades de análisis (Riches et al., 20II) y un aumento en la satisfacción con el trabajo que realizan (Beadle-Brown et al., 2012).

Totsika et al. (2008a) reflejan que las estrategias de apoyo activo que más utilizan los profesionales que participaron en su estudio son los planes de actividades diarias (realizados por el $70 \%$ ) y los planes de oportunidades (elaborados por el $65 \%$ de los trabajadores de apoyo directo). En el lado opuesto se encuentran los planes individuales o de enseñanza, los cuáles eran desconocidos por los propios profesionales antes de la puesta en marcha de la investigación.

Por último, y como aspecto positivo, cabe destacar que mientras que tradicionalmente los trabajadores tendían a prestar más atención a las personas con discapacidad intelectual que tenían más habilidades, la introducción del apoyo activo ha reportado mayores beneficios para las personas con discapacidades más severas (Smith et al., 2002), ya que tiene un impacto más significativo en sus vidas (Beadle-Brown et al., 20I4).

\section{- Resultados para las personas con discapacidad}

\section{Participación}

Uno de los aspectos que más se han analizado en la mayoría de investigaciones sobre el apoyo activo es la participación de la persona con discapacidad en diferentes actividades de su vida diaria como, por ejemplo, las actividades domésticas.

Todos los estudios que han analizado este constructo han visto un incremento a lo largo del proceso. En algunos casos ha sido, por ejemplo, del $35 \%$ (Jones et al., 200 Ia), del $7 \%$ (Stancliffe et al., 2008)... Mientras que en algunos de ellos el cambio en la participación en general ha sido significativo (Jones et al., I999, 200Ib; Mansell et al., 2002; Stancliffe et al., 2008; Koritsas et al., 2008; Chou et al., 20I I; Beadle-Brown et al., 20I2), para otros no ha llegado al nivel de significatividad (Smith et al., 2002).

Limitando esa participación al ámbito de las actividades domésticas, un alto número de los estudios examinados para la realización de esta revisión sustentan que ha aumentado significativamente tras la implantación del apoyo activo (Jones et al., I999, 2001a; Bradsahaw et al., 2004; Stancliffe et al., 2007, 2010; Riches et al., 20I I). Este incremento ha sido del I9,8\% al $37,2 \%$ en uno de los estudios desarrollados por Beadle-Brown et al. (20I2), I 2 meses después de haber introducido la metodología del AA en los diferentes hogares que compusieron su muestra.

Jones et al. (200Ia) recogen en su investigación un resultado que indica que las actividades sociales también han aumentado significativamente tras la formación de los profesionales en apoyo activo. Este mismo dato es plasmado por Beadle-Brown et al. (2012) en las conclusiones de su investigación.

Por lo tanto, parece claro que los estudios analizados ofrecen evidencias de que se ha producido un aumento en la participación de 
las personas con discapacidad (este incremento puede ser significativo o no) como fruto de la implantación en los hogares residenciales del AA. Además, se constata que el número y la variedad de actividades que se le ofrece a los residentes ha aumentado significativamente (Stancliffe et al., 2007, 2010).

\section{Interacción social}

Uno de los estudios realizados para comprobar los beneficios del apoyo activo varios años después de su introducción (Graham et al., 2013) refleja un cambio en el rol que desempeñan los residentes: un rol activo y más autónomo con un control mayor del entorno. A pesar de este avance, diversas investigaciones indican que, aun produciéndose esas mejoras, no se dan cambios significativos en la participación comunitaria de las personas con discapacidad (Beadle-Brown et al., 2012) ni en el contacto con familia y amigos (Stancliffe et al., 2007).

En relación a las elecciones, uno de los aspectos clave de la filosofía del AA, Stancliffe et al. (2007) han concluido en sus resultados que, aunque hayan incrementado, el cambio no es estadísticamente significativo. Por otra parte, el estudio de caso único realizado por Toogood et al. (2009) indica que la persona con discapacidad que ha sido examinada muestra un gran aumento en el período de interacciones con toma de decisiones del $\mathrm{I}_{3}, 5 \%$ al $83 \%$.

Koritsas et al. (2008) concluyen su estudio haciendo una aclaración sobre los ámbitos en los que se producen más y menos toma de decisiones: en el campo de las actividades domésticas y del uso de dinero los residentes realizan más elecciones, mientras que, en el lado contrario, aún queda trabajo que realizar en dominios como la salud, actividades sociales, acceso a la comunidad y relaciones personales. A pesar de ello, parece claro que el apoyo activo es una buena herramienta para mejorar la realización de elecciones de la persona con discapacidad intelectual (Chou $e t$ al., 20II).

\section{Depresión}

Poca es la información recogida en los estudios sobre los niveles de depresión de los residentes tras la adopción de la filosofía y estrategias del apoyo activo, pero aquellos estudios que los han analizado, recogen que se ha producido un descenso de los mismos (Stancliffe et al., 2007, 20IO), si bien en el estudio del 2007, Stancliffe et al. especifican que, a pesar de ser menor la puntuación en el área de la depresión, no hay una diferencia significativa.

\section{Conducta adaptativa y cambios de comportamiento}

Se han encontrado pequeños cambios en algunos estudios en relación a los cambios de comportamiento manifestados por las personas con discapacidad tras la implantación del apoyo activo (Jones et al., 200Ia; Stancliffe et al., 2007; Chou et al., 20I I), aunque no son significativos. En cambio, en el estudio de Stancliffe et al. (2010) sí se concluye que se ha producido un incremento significativo y en el de Bradshaw et al. (2004) se especifica que los cambios producidos se limitan a comportamientos leves como las estereotipias.

Respecto a la conducta adaptativa, uno de los trabajos desarrollados por Mansell et al. (20I3) refleja una mejor conducta adaptativa de su muestra analizada como resultado de unos niveles de participación altos. Por su parte, Beadle-Brown et al. (2008), señalan una reducción del 21 \% al 16\% de conductas repetitivas y, varios años después, los mismos investigadores exponen que el porcentaje de personas que muestran más de cinco comportamientos disruptivos ha disminuido, pero no significativamente (Beadle-Brown et al., 20I2). 


\section{Conclusiones}

Tras la lectura exhaustiva de los 24 artículos citados a lo largo de esta revisión, se han alcanzado las siguientes conclusiones:

- Parece claro que la formación en apoyo activo de los profesionales que ofrecen apoyo directo tiene una repercusión positiva en su forma de asistir a los residentes y, por ende, en la calidad de vida de las personas con discapacidad intelectual. A pesar de ello, la investigación llevada a cabo por Totsika et al. (2008a) abre la veda a una cuestión importante a tener en cuenta para futuras situaciones en las que se pretenda implantar el AA en un determinado servicio: "el entrenamiento que se realiza debería ser llevado a cabo por personas que conozcan bien a los residentes" en vez de formadores externos, con la finalidad de convertir ese proceso mecánico en una formación de mayor calidad y efectividad.

Por lo tanto, el uso de otras metodologías para la adquisición de conocimientos y estrategias sobre el AA, como el proceso empleado por Mansell et al. (2008) en su estudio exploratorio, en el que se proporcionaba una primera formación a los managers y, posteriormente estos formaban a los empleados, conseguiría una mayor incidencia en los profesionales ya que los managers, al conocer a los usuarios de los servicios, podrán aconsejarles sobre cómo realizar una asistencia más personalizada en función de sus necesidades de apoyo.

- A pesar de que la mayoría de los estudios analizados reflejan un aumento en la participación de los residentes y una mayor toma de decisiones, Graham et al. (2013) expone que una de las mayores preocupaciones emitidas por los profesionales que han participado en su estudio, ha sido la "falta de predictibilidad de las respuestas emitidas por las personas con discapacidad intelectual ante las nuevas experiencias". Si la persona nunca ha tenido la oportunidad de elegir qué es lo que quiere hacer en cada momento es posible que muestre como consecuencia conductas positivas como, por ejemplo, el aumento de su interés, pero también puede reaccionar con conductas negativas como comportamientos disruptivos fruto del estrés, del desconocimiento, de la desinformación o del miedo.

Por todo ello, es necesario que los profesionales, además de saber cómo dar ese apoyo, estén preparados y dispongan de técnicas y estrategias para atender este tipo de incidencias buscando siempre el bienestar de la persona con discapacidad.

- Otro de los aspectos que también preocupa al personal según los resultados emitidos por el estudio realizado por Koritsas et al. (2008) es la "falta de opciones que pueden proporcionar a las personas con discapacidad para que realicen elecciones". Fruto de esto son las pocas decisiones que las personas toman en las áreas de salud, actividades sociales, acceso a la comunidad y relaciones personales (Koritsas et al. 2008). Y es que, si los trabajadores solamente se pueden limitar a los recursos de los que dispone su institución, es muy complicado realizar cambios al respecto.

Por ello, es importante que los servicios se abran a la comunidad en la que se encuentran y, por su parte, esa comunidad debe de tener una actitud de colaboración. A su vez, el desarrollo y puesta en práctica de políticas sociales inclusivas serán la base para conseguir este objetivo.

- Tal y como se había reflejado en la tabla 4 , el "uso de instrumentos para recoger información acerca de los cambios acaecidos en la vida de los residentes y en la forma de prestar apoyo de los profesionales es muy variado". Se han utilizado desde herramientas estandarizadas o validadas por la comunidad científica hasta observaciones realizadas por los propios investigadores en 
períodos diferentes de tiempo. Esta última técnica ha sido la más utilizada en las investigaciones, concretamente, en I 6 de los 24 artículos analizados.

- En el estudio de Totsika et al. (2008a) se hace alusión a las "barreras existentes" para implantar la filosofía y las estrategias del apoyo activo. Estas son: la falta de ayudas por parte de los encargados de hacer la gestión del servicio (identificado por el $24 \%$ del personal), los cambios de humor que sufren los residentes (por el I9\%) y los pocos profesionales que están trabajando (19\%). A pesar de que estas son las barreras expuestas por los diez hogares que compusieron la muestra de este estudio en concreto, todas ellas pueden ser extrapoladas a cualquier organización ya que hacen referencias a problemas del día a día.

Por lo tanto, todas ellas han de ser consideradas y abordadas antes de iniciar cualquier estrategia para garantizar, sobre todo, el bienestar de las personas que reciben el apoyo.

- A pesar de las diferencias existentes en las investigaciones analizadas en esta revisión, la tendencia general indica que "el apoyo activo es una estrategia metodológica que proporciona beneficios en la vida diaria de la persona con discapacidad intelectual" (Beadle-Brown et al., 20I4). Esto concuerda con los resultados alcanzados por Hamelin y Sturmey (20II) que señalan, en su revisión sistemática, que la participación de los usuarios de los servicios varió, en general, del $66,7 \%$ al $78 \%$ durante la implantación del apoyo activo y del 8I \% al $93,3 \%$ en el seguimiento tras su puesta en marcha.

En esta misma revisión se calculó, tras el análisis de varios artículos de investigación, que la asistencia del personal varió del $60,6 \%$ al $85 \%$ en la fase de introducción de esta metodología mientras que, más adelante, aumentó del 8I \% al 9I \% en la etapa de seguimiento.
A su vez, estos estudios coinciden en señalar que el AA resulta más útil para aquellos residentes con mayores necesidades de apoyo ya que son los que requieren de una mayor y mejor asistencia (Jones et al., I999, 200Ia; Smith et al., 2002).

- $\quad$ Es destacable que "el roo\% de resultados encontrados a través del uso de los descriptores y de las bases de datos citados a lo largo de este artículo, son literatura inglesa". A su vez, los autores que firman los estudios suelen repetirse continuamente: personas como Mansell, Beadle-Brown, Stancliffe... participan en muchas de las investigaciones analizadas.

Por todo esto, se puede deducir que en España aún no se sabe mucho acerca de esta metodología tan beneficiosa para las personas con discapacidad intelectual severa (o con grandes necesidades de apoyo), por lo que su expansión dentro de la comunidad científica y del ámbito del trabajo directo podría suponer una mejora de la calidad de vida de estas personas.

Por último, se presenta un pequeño listado de claves para potenciar la incorporación de esta metodología con éxito en las entidades que trabajan con personas con discapacidad:

I. Todos los trabajadores del centro han de tener una actitud de colaboración, y una visión positiva del cambio.

2. La mejora de la calidad de vida de las personas con discapacidad ha de ser el primer principio que rija el trabajo de los profesionales.

3. La estructura de la entidad no ha de ser jerárquica, sino que ha de ser vertical, en la que no haya un marcado poder y donde todos los empleados tengan voz en el devenir de la institución.

4. Los profesionales del centro han de tener una correcta formación en apoyo activo tanto a nivel teórico como a nivel práctico.

5. El entrenamiento en esta metodología ha de ser promovido por personas que conozcan 
a los usuarios del servicio, para que éste sea más personal y con una mayor calidad.

6. Se ha de asumir la siguiente premisa: todas las personas, independientemente de su grado de discapacidad, pueden participar (de una forma u otra) en las actividades de su vida diaria.

7. Para potenciar esa participación se han de utilizar todos los recursos personales y materiales disponibles.

8. La participación de la persona con discapacidad en actividades de su entorno favorecerá un aumento en su índice de calidad de vida.

9. Los profesionales deben de tener una excelente coordinación para que la atención que reciban los usuarios del servicio sea de calidad, siendo tratados como personas con plenos derechos.

Io. Para evitar la falta de predictibilidad de las respuestas que puedan emitir las personas con discapacidad, se recomienda proporcionarles el mayor número de experiencias posibles.

I I. Los períodos de inactividad de la persona han de ser los que ella misma demande, y no los que decida el profesional.

I2. Una vez que la entidad ha asumido el apoyo activo como la metodología a utilizar diariamente, cada cierto tiempo ha de realizarse un seguimiento para ver si se están consiguiendo los objetivos propuestos y si se ha aumentado la participación de las personas con discapacidad. 
Association for Real Change (2009): Person centred active support. It ain't what we do it's "just" the way that we do it! Promoting active engagement (en línea). <http://arcuk.org.uk/ scotland/files/20 Io/I I/Person-centred-ActiveSupport-It-aint-what-we-do-its-just-the-waythat-we-do-it-Promoting-Active-Engagementpowerpoint.ppt $>$, acceso I 5 de febrero de 2016.

Beadle-Brown, J. et al. (20I4): "Practice leadership and active support in residential services for people with intellectual disabilities: an exploratory study". Journal of intellectual Disability Research, 58 (9): 838-850.

Beadle-Brown, J. et al. (2OI2): "Person- centred active support- Increasing choice, promoting independence and reducing challenging behavior". Journal of Applied Research in Intellectual Disabilities, 25 (4): 291-307.

Beadle-Brown, J. et al. (2008): “A better life: the implementation and effect of person-centred active support in the Avenues Trust". Tizard Learning Disability Review, I3 (4): I 5-24.

Bradshaw, J. et al. (2004): "Implementation and evaluation of active support". Journal of Applied Research in Intellectual Disabilities, I7 (3): I39-I 48 .

Cerolini, C. y Ferrie, J. (2005): Integrating recording systems within community residential units and ensuring residents have one individualized plan across all settings (en línea). <http://www.cds.med.usyd.edu.au/ cdsresearch-6o/publications-presentations/ doc_download/52-individualised-planning-andactive-support>, acceso I 5 de febrero de 2016 .

Chou, Y. et al. (20I I): "Outcome Evaluation of Active Support Training in Taiwan". Research in Developmental Disabilities, 32 (3): I I $30-$ II 36.

Emerson E. et al. (2000): “The quality and costs of community-based residential supports and residential campuses for people with severe and complex disabilities". Journal of Intellectual and Developmental Disability, 25 (4): 263-279.

Felce, D. et al. (2000): “The effectiveness of staff support: evaluating Active Support training using a conditional probability approach”. Research in Developmental Disability, 2 I (4): 243-255.

Fyffe, C. et al. (2008): "Initial investigation of organizational factors associated with the implementation of active support". Journal of Intellectual and Developmental Disability, 33 (3): 239, 246.

Graham, F. et al. (2013): "A more "normal" life: residents', family, staff and managers' experience of active support at a residential facility for people with physical and intellectual impairments". Journal of Intellectual and Developmental Disability, 38 (3): 256-264.

Hamelin, J. y Sturmey, P. (20I I): “Active support: a systematic review and evidence-based practice evaluation". Intellectual and Developmental Disabilities, 49 (3): I66-I7I.

Jones, E. et al. (201 I): Active support. A handbook for supporting people with learning disabilities to lead full lives, Bangor: Association for Real Change.

Jones, E. et al. (200Ia): "Evaluation of the dissemination of active support training in staffed community residences". American Journal of Applied Research in intellectual disabilities, 106 (4): 344-358.

Jones, E. et al. (200I b): "Evaluation of the dissemination of Active Support training and training trainers". Journal of Applied Research in Intellectual Disabilities, I4 (2): 79-99.

Jones, E. et al. (I999): "Opportunity and the promotion of activity among adults with severe intellectual disability living in community residences: the impact of training staff in active support". Journal of Intellectual Disability Research, 43 (3): I64-I 78. 
Koritsas, S. et al. (2008): "The effect of active support training on engagement, opportunities for choice, challenging behaviour and support needs". Journal of Intellectual and Developmental Disability, 33 (3): 247-256.

Mansell, J. et al. (2013): "Implementation of active support in Victoria, Australia: An exploratory study". Journal of Intellectual and Developmental Disability, 38 (I): 48-58.

Mansell, J. y Beadle-Brown, J. (20I2): Active support. Enabling and empowering people with intellectual disabilities, London: Jessica Kingsley.

Mansell, J. et al. (2008): "Effect of service structure and organization on staff care practices in small community homes for people with intellectual disabilities". Journal of Applied Research in Intellectual Disabilities, 2I (5): 398-4I3.

Mansell, J. et al. (2005): Person-Centred active support, Brighton: Pavilion Publishing.

Mansell, J. et al. (2002): "Engagement in meaningful activity and "active support" of people with intellectual disabilities in residential care". Research in Developmental Disabilities, 23 (5): 342-352.

Nui Galway. (2013): List of disability related academic journals (en línea). <http://www. nuigalway.ie/cdlp/documents/list_of_disability_ journals_cdlp_20I3.pdf $>$, acceso I 5 de enero de 2016.

Riches, V. et al. (2OI I): "Transforming staff practice through active support". Journal of Intellectual and Developmental Disability, 36 (3): I 56-I 66.

Smith, C. et al. (2002): "Responsiveness to staff support: evaluating the impact of individual characteristics on the effectiveness of active support training using a conditional probability approach". Journal of Intellectual Disability Research, 46 (8): 594-604.
Stancliffe, R. et al. (20I I): "Staffing numbers and active support: a case study". Tizard Learning Disability Review, I6 (3): 2I-30.

Stancliffe, R. et al. (2010): “Active support, participation and depression". Journal of Applied Research in Intellectual Disabilities, 23 (4): 3I2-32I.

Stancliffe, R. et al. (2008): "Staff behaviour and resident engagement before and after active support training". Journal of Intellectual and Developmental Disability, 33 (3): 257-270.

Stancliffe, R. et al. (2007): “Australian implementation and evaluation of Active Support”. Journal of Applied Research in Intellectual Disabilities, 20 (3): 2 I I-227.

Stancliffe, R. et al. (2005): Australian implementation and evaluation of active support, Sydney: Centre for Developmental Disability Studies

Toogood, S. et al. (2009): "Establishing a context to reduce challenging behaviour using procedures from active support: a clinical case example". Tizard Learning Disability Review, I4 (4): 29-36.

Totsika, V. et al. (2010): "The effect of active support interactive training on the daily lives of adults with an intellectual disability". Journal of Applied Research in Intellectual Disabilities, 23 (2): II 2-I 2 I.

Totsika, V. et al. (2008a): "Interactive training for active support: Perspectives from staff”. Journal of Intellectual and Developmental Disability, $33(3): 225-238$.

Totsika, V. et al. (2008b): “Active support. Developmental, evidence base and future directions". International Review of Research in Mental Retardation, 35:205-249. 